\title{
Body mass index in male patients with COPD: correlation with low attenuation areas on CT
}

\author{
E Ogawa, ${ }^{1}$ Y Nakano, ${ }^{2}$ T Ohara, ${ }^{1}$ S Muro, ${ }^{1}$ T Hirai, ${ }^{1}$ S Sato, ${ }^{1}$ H Sakai, ${ }^{3}$ M Tsukino, ${ }^{4}$ \\ D Kinose, ${ }^{1}$ M Nishioka, ${ }^{1}$ A Niimi, ${ }^{1}$ K Chin, ${ }^{1}$ P D Paré, ${ }^{5}$ M Mishima ${ }^{1}$
}

- Additional methods are published online only at http:// thorax.bmi.com/content/vol64/ issue1

${ }^{1}$ Department of Respiratory Medicine, Graduate School of Medicine, Kyoto University, Kyoto, Japan; ${ }^{2}$ Department of Respiratory Medicine, Shiga University of Medical Science, Otsu, Shiga, Japan;

${ }^{3}$ Department of Thoracic Surgery, Graduate School of Medicine, Kyoto University, Kyoto, Japan; ${ }^{4}$ Department of Respiratory Medicine, Hikone Municipal Hospital, Hikone, Shiga, Japan; ${ }^{5}$ The James Hogg iCAPTURE Centre and

Department of Medicine, St Paul's Hospital, University of British Colunbia, Vancouver, BC, Canada

Correspondence to:

Dr E Ogawa, Department of Respiratory Medicine, Kyoto University Hospital, 54 Shogoin, Kawahara-cho, Sakyo-ku, Kyoto, 606-8507, Japan; eogawa@ kuhp.kyoto-u.ac.jp

Received 18 February 2008 Accepted 30 August 2008

Published Online First

13 October 2008

\section{ABSTRACT}

Background: Chronic obstructive pulmonary disease (COPD) is characterised by the presence of airflow limitation caused by loss of lung elasticity and/or airway narrowing. The pathological hallmark of loss of lung elasticity is emphysema, and airway wall remodelling contributes to the airway narrowing. Using CT, these lesions can be assessed by measuring low attenuation areas (LAA) and airway wall thickness/luminal area, respectively. As previously reported, COPD can be divided into airway dominant, emphysema dominant and mixed phenotypes using CT. In this study, it is postulated that a patient's physique may be associated with the relative contribution of these lesions to airflow obstruction.

Methods: CT was used to evaluate emphysema and airway dimensions in 201 patients with COPD.

Emphysema was evaluated using percentage of LAA voxels (LAA\%) and airway lesion was estimated by percentage wall area (WA\%). Patients were divided into four phenotypes using LAA\% and WA\%.

Results: Body mass index (BMI) was significantly lower in the higher LAA\% phenotype (ie, emphysema dominant and mixed phenotypes). BMI correlated with LAA\% $(\rho=-0.557, p<0.0001)$ but not with WA\%. BMI was significantly lower in the emphysema dominant phenotype than in the airway dominant phenotype, while there was no difference in forced expiratory volume in $1 \mathrm{~s}$ \%predicted between the two.

Conclusion: A low BMl is associated with the presence of emphysema, but not with airway wall thickening, in male smokers who have COPD. These results support the concept of different COPD phenotypes and suggest that there may be different systemic manifestations of these phenotypes.

Chronic obstructive pulmonary disease (COPD) is characterised by the presence of airflow limitation caused by loss of lung elastic recoil and/or airway narrowing. ${ }^{1}$ Emphysema is the pathological lesion that correlates most closely with loss of lung elastic recoil, ${ }^{2}$ while the airway component is characterised by thickening and narrowing of membranous bronchioles. ${ }^{3}$ We have previously reported that the relative contributions of these processes to airflow obstruction in individual patients with COPD can be assessed by measuring low attenuation area (LAA) and airway wall thickness/luminal area using $\mathrm{CT}^{4}{ }^{4}$ Dividing patients with COPD into airway dominant, emphysema dominant and mixed phenotypes may aid in the study of the pathogenesis, in the assessment of pharmacological interventions and ultimately in the choice of patient specific therapy. ${ }^{6}$
There is increasing evidence that COPD is a systemic illness ${ }^{78}$ and low body weight is a prominent systemic manifestation. The cachexia associated with COPD was traditionally believed to be more prevalent among those whose airflow limitation was due to predominant emphysema and those who had a relatively maintained ventilatory drive (the "pink puffer" hypothesis)." The ability to make quantitative estimates of the degree of emphysema in individual patients allows a test of the longstanding hypothesis that emphysema predominant patients with COPD have a lower lean body mass.

We hypothesised that COPD phenotypes are associated with different patient physiques. In this study, we recruited 238 smokers and patients with COPD and divided them into four groups based on measurements of emphysema and airway dimensions using CT. Body mass index (BMI kg/ $\left.\mathrm{m}^{2}\right)$ was compared among the groups and the relationships between BMI and CT parameters were examined.

\section{METHODS}

\section{Subjects}

This is part of an ongoing COPD follow-up study in Kyoto University. A total of 239 smokers (219 patients with COPD from Kyoto University Hospital and 20 asymptomatic volunteers) were recruited. Results from a subset of these patients have been reported previously, ${ }^{4}{ }^{6}$ but the objectives of the present analyses are distinct from those addressed in the previous study. All asymptomatic volunteers were male, and there were 201 men and 18 women with COPD. Because we did not have enough women who had COPD to determine the influence of BMI in each gender, we excluded the 18 women from the analysis.

This study was a retrospective analysis of data collected in a prospective fashion and approved by the ethics committee of Kyoto University, and informed consent was obtained from each participant.

\section{Pulmonary function tests}

Forced expiratory volume in $1 \mathrm{~s}\left(\mathrm{FEV}_{1}\right)$ was expressed as \% predicted $\mathrm{FEV}_{1}$ (\% predicted) according to the prediction equations of the Japanese Society of Chest Disease.

\section{CT scans}

We used high resolution CT to quantify LAA and thin section helical CT to quantify airway 


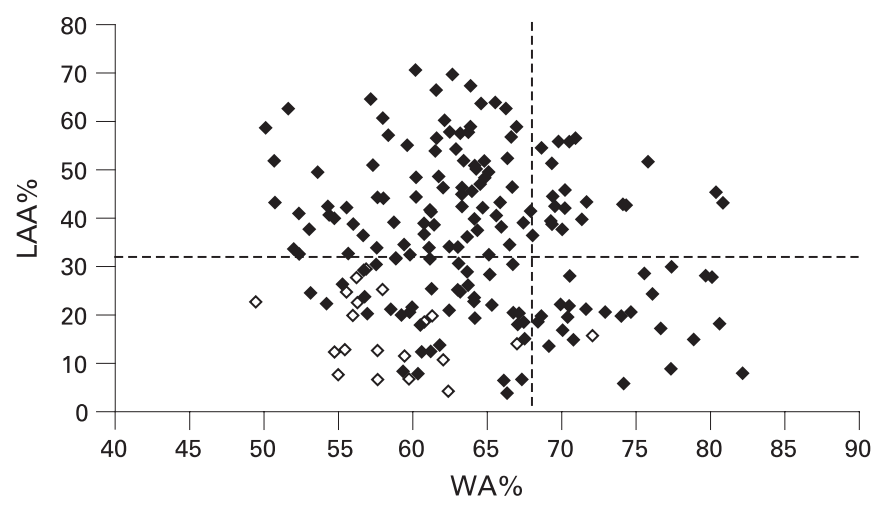

Figure 1 Relationship between percentage wall area (WA\%) and percentage of low attenuation voxels (LAA\%) in 165 patients with COPD (filled symbols) and 20 asymptomatic smokers (open symbols). Horizontal line shows the mean + 2SD of LAA\% of the asymptomatic smokers. Vertical line shows the mean + 2SD of WA\% of the asymptomatic smokers. Using these cut-off values, patients with COPD can be divided into phenotypes; normal by CT phenotype (low LAA\% and low WA\%), airway lesion dominant phenotype (high WA\% and low LAA\%), emphysema dominant phenotype (low WA\% and high LAA\%) and mixed phenotype (high WA\% and high LAA\%).

dimensions, as previously described. ${ }^{4}$ Both scans were performed in the supine position using the same CT scanner (XVigor; Toshiba, Tokyo, Japan).

\section{Analysis of low attenuation areas}

The percentage of low attenuation voxels (LAA\%) was calculated using a previously reported method, with $-960 \mathrm{HU}$ as the cut-off level. ${ }^{4} 10$

\section{Analysis of airway dimensions}

The dimensions of the right apical segmental bronchus were measured as previously described and the percentage wall area (WA\%) was used in the analyses. ${ }^{4}$ Some subjects $(n=36)$ whose CT scans were inadequate for determination of wall area were omitted from the airway analysis.

Table 1 Anthropometric and pulmonary function data for the 221 subjects

\begin{tabular}{lcc}
\hline & Volunteers $(\mathbf{n}=\mathbf{2 0})$ & COPD $(\mathbf{n}=\mathbf{2 0 1})$ \\
\hline Age (years) & $64(16)$ & $71(7)^{* *}$ \\
Smoking Index (pack-years) & $57(44)$ & $71(38)$ \\
Height $(\mathrm{cm})$ & $166(7)$ & $163(6)^{*}$ \\
Weight $(\mathrm{kg})$ & $64(7)$ & $57(9)^{* *}$ \\
BMI $\left(\mathrm{kg} / \mathrm{m}^{2}\right)$ & $23.2(2.2)$ & $21.4(2.9)^{* *}$ \\
BSA $\left(\mathrm{m}^{2}\right)$ & $1.71(0.13)$ & $1.60(0.13)^{* *}$ \\
FVC $(\%$ predicted) & $95(18)$ & $72(18)^{* *}$ \\
FEV $(\% p r e d i c t e d)$ & $96(21)$ & $46(18)^{* *}$ \\
FEV $/$ FVC (\%) & $73(11)$ & $43(12)^{* *}$ \\
RV/TLC $(\%)$ & $34(9)$ & $47(9)^{* *}$ \\
Tlco $(\mathrm{mmol} / \mathrm{min} / \mathrm{kPa} / \mathrm{l})$ & $1.50(0.29)$ & $1.04(0.43)^{* *}$ \\
\hline
\end{tabular}

Values are mean (SD).

Smoking Index $=$ (the average number of packs smoked/day (20 cigarettes/pack $) \times$ years of smoking).

$\mathrm{BMI}$, body mass index; BSA, body surface area; COPD, chronic obstructive pulmonary disease; $\mathrm{FEV}_{1}$, forced expiratory volume in $1 \mathrm{~s}$; FVC, forced vital capacity; RV, residual volume; TLC, total lung capacity; TIco, carbon monoxide transfer coefficient.

${ }^{*} \mathrm{p}<0.05$, ${ }^{* *} \mathrm{p}<0.01$ compared with volunteers.

\section{COPD phenotypes}

As previously reported, patients with COPD can be divided into four groups based on the CT measurements of LAA\% and WA\%. ${ }^{6}$ The four groups were: normal by CT (NCT; low LAA\% and low WA\%), airway dominant (AD; low $\mathrm{LAA} \%$ and high WA\%), emphysema dominant (ED; high LAA\% and low WA\%) and mixed (high LAA\% and high WA\%) phenotypes, respectively. Figure 1 shows the distribution of all subjects in relation to $\mathrm{LAA} \%$ and $\mathrm{WA} \%$.

\section{Analysis of subcutaneous fat}

To determine whether there were any other parameters of the patient's physique which correlated with BMI and with CT parameters, we measured subcutaneous fat mass. As the CT scans were of the thorax, we first validated whether subcutaneous fat at this location reflected the more commonly used abdominal fat mass.

\section{Serum markers and LAA\%}

Among 201 male patients with COPD, we were able to obtain serum markers of nutritional status in 81 .

\section{Statistical method}

Results are expressed as mean (SD). The Kruskal-Wallis test was used to compare groups and then the Mann-Whitney $U$ test was used to compare two groups with Bonferroni correction. Spearman's rank correlation coefficient was used to evaluate relationships between BMI, LAA\% and the CT parameters and pulmonary function tests. A value of $p<0.05$ was considered significant.

Detailed methods are described in the supplementary material available online only.

\section{RESULTS}

\section{Patient characteristics and phenotypes}

The characteristics of the 20 male volunteers and the 201 male patients with COPD are shown in table 1. Patients with COPD were older and had a lower BMI and body surface area (BSA) and, by design, substantially obstructed lung function. There was no difference in cigarette smoke exposure between the two groups.

\section{Comparison of data among phenotypes}

As shown in table 2, there were no differences in age, smoking index or height among the four phenotypes. However, body weight and BMI were significantly lower in the ED and mixed phenotypes compared with the NCT and/or the AD phenotypes. BSA was significantly lower in the ED and mixed phenotypes. There was no difference in residual volume/total lung capacity (TLC) (\%) among the four phenotypes. There was no difference in $\mathrm{FEV}_{1}$ (\% predicted) between the $\mathrm{AD}$ and $\mathrm{ED}$ phenotypes. As previously reported, carbon monoxide transfer coefficient (Tlco) was significantly lower in the phenotypes that had higher LAA\%, the ED and mixed phenotypes, than in the NCT and $\mathrm{AD}$ phenotypes. ${ }^{6}$ We reanalysed the data using a Tlco value of $\angle 2$ SD below the mean value for the non-COPD smokers instead of LAA\% as the definition of emphysema and using the median values of $\mathrm{LAA} \%$ and $\mathrm{WA} \%$ as cut-off criteria. Although these analyses altered the number of subjects in the different groups, the main conclusions were unchanged; the ED group still had the lowest BMI $(p<0.0001)$ (data not shown). 
Table 2 Anthropometric and pulmonary function data and CT measured parameters in each of the COPD subgroups $(n=165)$

\begin{tabular}{|c|c|c|c|c|}
\hline & NCT $(n=41)$ & $A D(n=24)$ & $E D(n=81)$ & Mixed $(n=19)$ \\
\hline Age (years) & $70(7)$ & $70(9)$ & $71(7)$ & $70(6)$ \\
\hline Smoking Index (pack-years) & $70(44)$ & $72(44)$ & $69(27)$ & $84(55)$ \\
\hline Height (cm) & $163(7)$ & $161(7)$ & $163(6)$ & $159(6)$ \\
\hline Weight (kg) & $61(9)$ & $60(9)$ & $54(8)^{*} \dagger$ & $53(6)^{*}$ \\
\hline BMI $\left(\mathrm{kg} / \mathrm{m}^{2}\right)$ & $22.9(2.1)$ & $23.0(2.6)$ & $20.2(2.8)^{*} \dagger$ & $21.0(2.8)^{*}$ \\
\hline BSA $\left(m^{2}\right)$ & $1.65(0.15)$ & $1.61(0.14)$ & $1.58(0.12)$ & $1.54(0.10)$ \\
\hline FVC (\%predicted) & $72(17)$ & $64(17)$ & $76(19) \dagger$ & $68(14)$ \\
\hline $\mathrm{FEV}_{1}$ (\%predicted) & $54(18)$ & $44(17)$ & $43(18)^{*}$ & $36(13)^{*}$ \\
\hline $\mathrm{FEV}_{1} / \mathrm{FVC}(\%)$ & $50(12)$ & $48(11)$ & $38(10)^{*} \dagger$ & $37(10)^{*} \dagger$ \\
\hline RV/TLC (\%) & $45(9)$ & $50(11)$ & $48(9)$ & $51(7)$ \\
\hline TIco (mmol/min/kPa/l) & $1.27(0.39)$ & $1.52(0.45)$ & $0.85(0.30)^{*} \dagger$ & $1.00(0.33)^{*}+$ \\
\hline WA $\%(\%)$ & $61.9(4.0)$ & $74.2(4.2)^{*}$ & $61.0(4.7) \dagger$ & $71.8(3.7)^{*} \star$ \\
\hline LAA $\%(\%)$ & $21.1(7.5)$ & $19.6(6.5)$ & $47.2(10.2)^{*} \dagger$ & $45.7(6.5)^{*} \dagger$ \\
\hline
\end{tabular}

Values are mean (SD).

Smoking Index $=$ (the average number of packs smoked/day (20 cigarettes/pack) $\times$ years of smoking).

$A D$, airway dominant; $B M I$, body mass index; $B S A$, body surface area; COPD, chronic obstructive pulmonary disease; $E D$ emphysema dominant; $\mathrm{FEV}_{1}$, forced expiratory volume in $1 \mathrm{~s}$; $F V C$, forced vital capacity; LAA\%, percentage of low attenuation area; NCT, normal by CT; RV, residual volume; TLC, total lung capacity; TIco, carbon monoxide transfer coefficient; WA\%, percentage of the wall area.

${ }^{*} p<0.05$ versus NCT; $\uparrow p<0.05$ versus $A D ; t p<0.05$ versus ED.

A significant relationship of BMI with LAA\% but not with WA\% Because BMI was significantly lower in the ED and mixed phenotypes, we next analysed the relationship between BMI and LAA $\%(n=201)$. There was an inverse relationship between BMI and LAA $\%(\rho=-0.557, p<0.0001)$ (table 3$)$. LAA\% was also significantly related to body weight $(\rho=-0.456, p<0.0001)$ and BSA $(\rho=-0.266, p=0.0001)$ (table 3). On the other hand, WA $\%$ had no significant relationship with BMI, weight or BSA (table 3) $(\mathrm{n}=165)$. The same results were apparent when these relationships were examined within phenotypic subgroups; LAA\% was significantly related to $\mathrm{BMI}$ in the $\mathrm{AD}, \mathrm{ED}$ and mixed groups but WA\% was not related to BMI in any of the groups (data not shown). Table 3 also shows Spearman's rank correlation coefficients for the relationship of BMI, LAA\% and WA\% with additional patient characteristics. LAA\% and TlCo were highly correlated $(\rho=-0.642, p<0.0001)$. BMI, LAA $\%$ and WA $\%$ were significantly related to $\mathrm{FEV}_{1}$ (\% predicted).

Although $\mathrm{FEV}_{1}$ (\% predicted) correlated with BMI and LAA\% (table 3), there was no difference in mean $\mathrm{FEV}_{1}$ (\% predicted) between subjects who had the $\mathrm{AD}$ and $\mathrm{ED}$ phenotypes (table 2).
However, BMI was significantly lower in those with the ED phenotype.

\section{Measurement of subcutaneous fat mass and the relationship between LAA\% and subcutaneous fat mass}

As described in the methods section, we tested whether the subcutaneous fat mass at the level of the umbilicus correlated with fat mass in other locations. These results show that fat area measured in the thoracic region is an adequate surrogate for subcutaneous fat at the umbilical level. Among the 201 patients with COPD, we were able to measure the fat area at the thoracic level in 137 but could not measure it in 64 because a portion of the subcutaneous fat was beyond the field of view of the scan. The area of subcutaneous fat mass correlated with BMI $(\rho=0.736, p<0.0001)$ and with LAA\% $(\rho=-0.307$, $\mathrm{p}<0.0001)$

\section{Serum markers and LAA\%}

In 81 patients with COPD, none of the serum markers we measured (total protein $(7.0(0.4) \mathrm{g} / \mathrm{dl})$, albumin $(4.3(0.3) \mathrm{g} / \mathrm{dl})$,

Table 3 Spearman's rank correlation coefficient ( $\rho / p$ values) for BMI, LAA\% and WA\% in patients with COPD

\begin{tabular}{|c|c|c|c|c|c|c|}
\hline & \multicolumn{2}{|l|}{ BMI } & \multicolumn{2}{|l|}{ LAA $\%$} & \multicolumn{2}{|l|}{ WA $\%$} \\
\hline & $\rho$ & p Value & $\rho$ & p Value & $\rho$ & p Value \\
\hline Age (years) & 0.040 & 0.5721 & 0.050 & 0.4813 & -0.022 & 0.7802 \\
\hline Smoking Index (pack-years) & -0.027 & 0.0701 & 0.092 & 0.1940 & -0.012 & 0.8772 \\
\hline Height $(\mathrm{cm})$ & - & - & 0.092 & 0.1958 & -0.225 & 0.0037 \\
\hline Weight (kg) & - & - & -0.456 & $<0.0001$ & -0.017 & 0.8255 \\
\hline BSA $\left(m^{2}\right)$ & - & - & -0.266 & 0.0001 & -0.094 & 0.2303 \\
\hline $\mathrm{FEV}_{1}(\mathrm{l} / \mathrm{s})$ & 0.280 & $<0.0001$ & -0.318 & $<0.0001$ & -0.164 & 0.0356 \\
\hline $\mathrm{FEV}_{1}$ (\%predicted) & 0.315 & $<0.0001$ & -0.306 & $<0.0001$ & -0.176 & 0.0241 \\
\hline $\mathrm{FEV}_{1} / \mathrm{FVC}(\%)$ & 0.384 & $<0.0001$ & -0.495 & $<0.0001$ & -0.009 & 0.9098 \\
\hline RV/TLC (\%) & -0.195 & 0.0057 & 0.170 & 0.0159 & 0.262 & 0.0007 \\
\hline Tlco (mmol/min/kPa/l) & 0.337 & $<0.0001$ & -0.642 & $<0.0001$ & 0.260 & 0.0007 \\
\hline WA $\%(\%)$ & 0.111 & 0.156 & -0.183 & 0.0019 & - & - \\
\hline LAA $\%(\%)$ & -0.557 & $<0.0001$ & - & - & - & - \\
\hline
\end{tabular}

Smoking Index $=$ (the average number of packs smoked/day (20 cigarettes/pack) $\times$ years of smoking).

$\mathrm{BMI}$, body mass index; BSA, body surface area; FVC, forced vital capacity; FEV , forced expiratory volume in $1 \mathrm{~s}$; LAA\%,

percentage of low attenuation area; RV, residual volume; TLC, total lung capacity; TIco, carbon monoxide transfer coefficient, bold text indicate significant correlations; WA\%, percentage of wall area. 
cholinesterase (303 (71) IU/l), triglycerides (124 (73) mg/dl), total cholesterol $(198(38) \mathrm{mg} / \mathrm{dl})$ or $\mathrm{C}$ reactive protein $(0.3$ (1.0) $\mathrm{mg} / \mathrm{dl})$ ) correlated with BMI or LAA\%.

\section{DISCUSSION}

COPD is defined functionally by a decrease in maximal expiratory flow from the lung. The decrease in expiratory flow, as well as the other functional hallmarks of COPD (hyperinflation, gas trapping and decreased gas transfer), can be caused by destruction of the lung parenchyma and/or heterogeneous small airway remodelling and narrowing. CT is now recognised as a valuable tool to evaluate both lung parenchymal integrity and airway dimensions. ${ }^{4-6}{ }^{10-16}$ Individual subjects with COPD have different combinations of parenchymal and airway lesions and in previous studies we have proposed the use of CT scanning to separate these phenotypes. ${ }^{4-6}$ In the present study, we investigated whether subjects with predominant emphysema (LAA\%) differed from those with predominant airway narrowing (WA\%) with respect to body habitus. We found that individuals who had the predominant LAA\% phenotype had a lower BMI. BMI correlated with LAA\% but not with WA\%.

There are several reports which support our data that BMI is lower in predominantly emphysematous COPD patients evaluated by CT data. ${ }^{17-19}$ However, in most of the previous reports, emphysema was evaluated semiquantitatively. Miniati et al evaluated emphysematous lesions quantitatively but on chest radiographs not CT scans. ${ }^{20}$ On the other hand, there are reports on the relationship between airway wall thickness and BMI. Fujimoto et al used a visual estimate of airway wall thickness and found that patients with COPD who had an airway dominant phenotype had a higher BMI than those with an emphysema dominant or mixed phenotype. ${ }^{21}$ Lee et al evaluated LAA\% and WA\% quantitatively and demonstrated that LAA \% was negatively correlated with BMI, which is consistent with our results. But they also showed a positive correlation between WA\% and BMI. ${ }^{22}$ However, their analysis was done using relatively small numbers of patients $(n=34)$.

Our finding of an association of an emphysema predominant phenotype in patients with COPD and a lower BMI is in keeping with the longstanding observation that a subset of patients with COPD are "pink puffers"..$^{-9}$ This traditional clinical designation was used to characterise patients with COPD who had predominant emphysema, relatively preserved ventilatory drive and a low BMI. As in any previous studies of these COPD sub-phenotypes, this study does not allow us to determine whether the decreased BMI is secondary to the emphysematous process which results in increased LAA or whether the decreased BMI contributes to the emphysema predominant phenotype. There are lines of evidence to support either causal pathway.

Low BMI could be a consequence of COPD. Basal metabolic rate is increased in moderate to severe COPD. ${ }^{23}$ Decramer et al have proposed that the pathogenesis of nutritional depletion in COPD is high energy expenditure and low energy intake. ${ }^{24}$ Importantly, low BMI is one of the independent predictors for mortality in patients with COPD.$^{25}$ These observations have led to a number of trials to intervene with nutritional support for patients with COPD. ${ }^{26-28}$ Our findings support these associations in that there was a significant relationship between the severity of $\mathrm{COPD}$, as assessed by $\mathrm{FEV}_{1} \%$ predicted, and $\mathrm{BMI}$

Could it be that lean smokers are more likely to develop parenchymal destruction as the primary component of their COPD? There is a substantial literature to support the concept that nutritional depletion can contribute to the development of emphysema. In an autopsy study performed in the Warsaw Ghetto during World War II, a high percentage of young people who died of malnutrition showed evidence of emphysema. ${ }^{29}$ Animal models of starvation induced "emphysema" have supported a relationship. Severe calorie restriction leads to decreased production of surfactant ${ }^{30}$ and a reduction in the number of alveoli with a corresponding increase in alveolar volume and decrease in lung surface area. ${ }^{31}$ These reports do not directly implicate tissue destruction as the pathogenetic mechanism for these changes and therefore the lesions are usually called "emphysema-like." Coxson et al have recently reported similar results in patients who have anorexia nervosa. ${ }^{32}$ They used a quantitative CT method and found that CT lung density was significantly lower in anorexic patients than in the age matched control group. They also reported significant relationships between BMI and the CT estimate of "emphysema" and between the extent of low attenuation areas and diffusing capacity. ${ }^{32}$

In this study, we further investigated the relationship of LAA \% with subcutaneous fat mass and serum parameters of nutrition. BMI correlated with thoracic subcutaneous fat mass, and subcutaneous fat mass was significantly related to LAA\%. Hypoalbuminaemia has been reported in patients with COPD compared with other hypoxic lung diseases and age matched controls. ${ }^{33}$ The authors of the study have suggested that low albumin was explained by malnutrition and/or chronic inflammation. However, neither BMI nor LAA\% was related to serum markers of nutrition or $C$ reactive protein, in this study. The smaller sample size for this comparison may have explained the failure to find relationships. Cachexia in patients with COPD may be caused not only by malnutrition but also by systemic inflammation.

It is now generally recognised that COPD is associated with systemic manifestations. ${ }^{72}$ Patients with COPD have a significant increase in the circulating level of $\mathrm{C}$ reactive protein ${ }^{34}$ and evidence of increased risk for coronary vascular disease. ${ }^{35}$ Levels of soluble tumour necrosis factor receptor have been reported to be significantly increased in the circulating blood of patients with COPD, and patients with emphysema have a lower lean body mass and lower levels of leptin than subjects with chronic bronchitis. ${ }^{36}$ On the other hand, Cohen et al found no significant difference in serum leptin levels between emphysematous and healthy subjects ${ }^{37}$ and suggested that "hypermetabolism" in emphysematous subjects is not sufficient to lead to weight loss. ${ }^{37}$ The mechanism of body weight loss in patients with COPD is still unclear. It is possible that a vicious cycle is set up in some COPD patients with emphysema leading to weight loss and weight loss contributing to emphysema; however, this does not explain why there is a subset of patients with COPD who have a well preserved BMI. Whatever the mechanism, the relationship of emphysema and reduced BMI has spawned a number of studies designed to test whether nutritional support can influence disease severity in emphysema. ${ }^{27}{ }^{28}$ A recent meta-analysis of randomised controlled trials revealed that nutritional support was not associated with improvements in anthropometric measures or functional exercise capacity among patients with stable COPD. ${ }^{26}$

There are two technical factors which could have influenced our results and potentially contributed to the relationship between BMI and LAA\% that we observed. One factor relates to the physics of CT scanning. Individuals with a higher BMI will have thicker chest walls and therefore less $x$ ray energy will reach the CT detectors. However, this potential artefact would tend to work in the opposite direction to the result that we 
observed. Recently, Yuan et al examined the influence of decreased radiation dose on LAA and found that decreased radiation dose causes an apparent increase in LAA by leading to a wider distribution of voxel density. ${ }^{38}$ This potential artefact may underestimate the results of this study but strengthen our conclusions. Madani et al examined how radiation dose and section thickness influence CT quantification of pulmonary emphysema. ${ }^{15}$ They found that the LAA\% at $20 \mathrm{mAs}$ predicted the macroscopic and microscopic emphysema as accurately as the LAA \% at $120 \mathrm{mAs}$ when the threshold was $-960 \mathrm{HU}$. Thus we do not think the patient's physique could result in artefacts in the measurement of LAA.

The other technical issue that could influence our results is the lung volume achieved during the CT scan. Failure to inspire to the same extent in heavier individuals could have reduced their apparent LAA\%. Subjects are asked to inspire fully during the acquisition of CT scan data but the volume that they achieve is not TLC. It is well known that the maximal volume achieved is less in the supine position and that the effect of supine position on reducing true TLC is greater in obese individuals. Although our subjects were not truly obese (19/201 had a BMI of 25-29.9 which is considered overweight, and none had a BMI $>30$, which is the accepted threshold for obesity according to the guidelines of the National Institute of Health), we considered this as a possible source of error and therefore we attempted to estimate the relative lung volume subjects had achieved in the scanner by comparing the ratio of the area of lung segmented from the chest wall in the three CT slices to measured TLC. We reasoned that if heavier individuals failed to achieve the same lung volume as those with a lower BMI, they would have a lower ratio of lung area to TLC. However, there was no relationship between this ratio and BMI $(p=0.5119)$. Thus we do not believe that differential lung expansion during the CT scans can explain our results.

We used $-960 \mathrm{HU}$ as the threshold for measuring LAA\%. As previously described, we chose this threshold because the mean $-2 \mathrm{SD}$ CT number in the lungs of the volunteers was approximately $-960 \mathrm{HU} \cdot{ }^{10}$ Gevenois et al investigated the relationship between macroscopic and microscopic lung morphometry and CT indices, and found that $-950 \mathrm{HU}$ was suitable for evaluating emphysema using thin section CT. ${ }^{14}$ More recently, Madani et al have used a helical thin section multidetector row CT scanner and suggested that $-960 \mathrm{HU}$ and $-970 \mathrm{HU}$ were the most appropriate thresholds for quantifying emphysema. ${ }^{16}$

We did find a negative correlation between WA\% and height. To our knowledge, there are no previous reports of the relationship between airway wall thickness and height. Since height is closely related to lung volume, this relationship probably reflects the fact that taller individuals have larger lungs and larger airways. There is an inverse relationship between airway size and relative wall area within a subject's lung ${ }^{39}$ and a similar between subject relationship is the likely explanation for this finding.

There are some limitations to our study. Firstly, we used only three CT slices for the evaluation of emphysema despite heterogeneously distributed emphysema. The main reason for this limited number of slices was to reduce the total radiation dose for the subjects. In previous studies we have evaluated the number of slices and found that three slices were sufficient for the evaluation of emphysema. ${ }^{10}$

Secondly, we used only one large airway for the evaluation of airway dimensions. There was no relationship between BMI and airway dimensions measured by CT. We measured the dimensions of a large airway, the apical segmental bronchus to the right upper lobe, even though it is well known that the site of major airway obstruction in COPD is the small airways. ${ }^{3}$ However, we have previously shown that the dimensions of this relatively large bronchus serve as a useful surrogate for small airway remodelling, and WA\% in this airway is independently related to airflow obstruction. ${ }^{5}$ As an added validation, we selected 45 patients in whom we were able to measure the WA\% of another airway, the posterior basal segmental bronchus $\left(\mathrm{B}^{10}\right)$, and found that WA\% of the apical segmental bronchus $\left(\mathrm{B}^{1}\right)$ correlated significantly with WA\% of $\mathrm{B}^{10}$ $(\rho=0.431, \quad p=0.0006)$. However, there was no relation between WA\% of $\mathrm{B}^{10}$ and LAA\%. Despite this however, it is possible that the lack of a significant relationship between BMI and WA\% (a positive relationship is suggested) may be due to the relatively imprecise and indirect estimate of small airway wall area.

An additional potential limitation is the NCT group who do not fit into the $\mathrm{ED}, \mathrm{AD}$ or mixed phenotype. We can only speculate as to the mechanism underlying the obstruction in these patients. It may be that they have airway wall remodelling confined to the small airways beyond the resolution of the CT scan. Additionally, they may have diffuse panacinar emphysema on a scale that does not result in a reduction of density beyond the threshold in the voxels that we sample.

Fourthly, we only analysed the data from male patients. As gender difference may have a substantial influence in COPD, ${ }^{40}$ further investigation is needed.

A final potential limitation of the analysis is the potential for type I error related to the multiple comparisons that were made. Although we used a Bonferroni correction for each particular comparison, there is still a chance of false positive association. However, many of the parameters we compared (eg, weight, BSA, BMI) were correlated so that the true number of independent tests was not as great as if they were unrelated. The consistency of the results across comparisons is also supportive of our conclusions.

In conclusion, we found a significant inverse relationship between BMI and the extent of emphysema on CT scan in the lungs of male smokers. It is unclear if emphysema predisposes to weight loss among patients who develop COPD or whether low body weight contributes to the development of emphysema. A test of the direction of the relationship might be answered by an interventional study examining whether nutritional support could attenuate the progression of emphysematous lesions. As this was a retrospective analysis, further prospective studies using recently described methods to assess volumetric CT data are needed to clarify the relationship between body weight and the destruction of lung parenchyma.

Funding: This study was supported partly by a grant from the Respiratory Failure Research Group from the Ministry of Health, Labour and Welfare, Japan, and partly by a Grant-in-Aid for Scientific Research (C) (No.18590847) from the Japan Society for the Promotion of Science (JSPS).

\section{Competing interests: None.}

Ethics approval: This study was a retrospective analysis of data collected in a prospective fashion and approved by the ethics committee of Kyoto University.

\section{REFERENCES}

1. Bethesda M. Global strategy for the diagnosis, management, and prevention of chronic obstructive pulmonary disease updated. 2006. http://www.goldcopd.com (accessed 5 November 2008).

2. Paré PD, Brooks LA, Bates J, et al. Exponential analysis of the lung pressure-volume curve as a predictor of pulmonary emphysema. Am Rev Respir Dis 1982;126:54-61. 
3. Hogg JC, Chu F, Utokaparch S, et al. The nature of small-airway obstruction in chronic obstructive pulmonary disease. N Engl J Med 2004;350:2645-53.

4. Nakano Y, Muro S, Sakai H, et al. Computed tomographic measurements of airway dimensions and emphysema in smokers. Correlation with lung function. Am J Respir Crit Care Med 2000;162:1102-8.

5. Nakano Y, Wong JC, de Jong PA, et al. The prediction of small airway dimensions using computed tomography. Am J Respir Crit Care Med 2005;171:142-6.

6. Nakano Y, Müller NL, King GG, et al. Quantitative assessment of airway remodeling using high-resolution CT. Chest 2002;122:271S-5S.

7. Agusti AG, Noguera A, Sauleda J, et al. Systemic effects of chronic obstructive pulmonary disease. Eur Respir J 2003;21:347-60.

8. Schols AM. Nutrition in chronic obstructive pulmonary disease. Curr Opin Pulm Med 2000;6:110-15.

9. Annotator. Blue bloater: pink puffer. Br Med J 1968;2:677.

10. Mishima M, Hirai T, Jin Z, et al. Standardization of low attenuation area versus total lung area in chest $\mathrm{X}$-ray $\mathrm{CT}$ as an indicator of chronic pulmonary emphysema. Front Med Biol Eng 1997;8:79-86.

11. Berger $\mathbf{P}$, Perot $\mathrm{V}$, Desbarats $\mathbf{P}$, et al. Airway wall thickness in cigarette smokers: quantitative thin-section CT assessment. Radiology 2005;235:1055-64.

12. Montaudon M, Berger P, Cangini-Sacher A, et al. Bronchial measurement with three-dimensional quantitative thin-section CT in patients with cystic fibrosis. Radiology 2007:242:573-81.

13. Orlandi I, Moroni C, Camiciottoli G, et al. Chronic obstructive pulmonary disease: thin-section CT measurement of airway wall thickness and lung attenuation. Radiology 2005;234:604-10

14. Gevenois PA, De Vuyst $\mathrm{P}$, de Maertelaer $\mathrm{V}$, et al. Comparison of computed density and microscopic morphometry in pulmonary emphysema. Am J Respir Crit Care Med 1996;154:187-92.

15. Madani A, De Maertelaer V, Zanen J, et al. Pulmonary emphysema: radiation dose and section thickness at multidetector CT quantification-comparison with macroscopic and microscopic morphometry. Radiology 2007;243:250-7.

16. Madani A, Zanen J, de Maertelaer V, et al. Pulmonary emphysema: objective quantification at multi-detector row CT_-comparison with macroscopic and microscopic morphometry. Radiology 2006;238:1036-43.

17. Kitaguchi Y, Fujimoto K, Kubo K, et al. Characteristics of COPD phenotypes classified according to the findings of HRCT. Respir Med 2006;100:1742-52.

18. Makita H, Nasuhara $Y$, Nagai K, et al. Characterisation of phenotypes based on severity of emphysema in chronic obstructive pulmonary disease. Thorax 2007;62:932-7.

19. Pistolesi M, Camiciottoli G, Paoletti M, et al. Identification of a predominant COPD phenotype in clinical practice. Respir Med 2008;102:367-76.

20. Miniati M, Monti S, Stolk J, et al. Value of chest radiography in phenotyping chronic obstructive pulmonary disease. Eur Respir J 2008;31:509-15.

21. Fujimoto K, Kitaguchi $Y$, Kubo $K$, et al. Clinical analysis of chronic obstructive pulmonary disease phenotypes classified using high-resolution computed tomography. Respirology 2006;11:731-40.
22. Lee $\mathbf{Y K}$, Oh YM, Lee $\mathrm{JH}$, et al. Quantitative assessment of emphysema, air trapping, and airway thickening on computed tomography. Lung 2008;186:157-65.

23. Hugli $\mathbf{0}$, Schutz $Y$, Fitting JW. The daily energy expenditure in stable chronic obstructive pulmonary disease. Am J Respir Crit Care Med 1996;153:294-300.

24. Decramer M, De Benedetto F, Del Ponte A, et al. Systemic effects of COPD. Respir Med 2005;99:S3-10.

25. Celli BR, Cote CG, Marin JM, et al. The body-mass index, airflow obstruction, dyspnea, and exercise capacity index in chronic obstructive pulmonary disease. N Engl J Med 2004;350:1005-12.

26. Ferreira IM, Brooks D, Lacasse $Y$, et al. Nutritional support for individuals with COPD: a meta-analysis. Chest 2000;117:672-8.

27. Steiner MC, Barton RL, Singh SJ, et al. Nutritional enhancement of exercise performance in chronic obstructive pulmonary disease: a randomized controlled trial. Thorax 2003;58:745-51.

28. Wilson D0, Rogers RM, Sanders MH, et al. Nutritional intervention in malnourished patients with emphysema. Am Rev Respir Dis 1986;134:672-7.

29. Stein JFH. Pathological anatomy of hunger disease. In: Winick M, ed. Hunger disease: studies by the Jewish physicians in theWarsaw Ghetto: current concepts in nutrition. New York: John Wiley \& Sons, 1979.

30. Gail DB, Massaro GD, Massaro D. Influence of fasting on the lung. J Appl Physiol 1977:42:88-92.

31. Harkema JR, Mauderly JL, Gregory RE, et al. A comparison of starvation and elastase models of emphysema in the rat. Am Rev Respir Dis 1984;129:584-91.

32. Coxson HO, Chan IH, Mayo JR, et al. Early emphysema in patients with anorexia nervosa. Am J Respir Crit Care Med 2004;170:748-52.

33. Cano NJ, Roth H, Court-Ortune I, et al. Nutritional depletion in patients on long-term oxygen therapy and/or home mechanical ventilation. Eur Respir J 2002;20:30-7.

34. Man SF, Connett JE, Anthonisen NR, et al. C-reactive protein and mortality in mild to moderate chronic obstructive pulmonary disease. Thorax 2006;61:849-53.

35. Sin DD, Man SF. Chronic obstructive pulmonary disease: a novel risk factor for cardiovascular disease. Can J Physiol Pharmacol 2005;83:8-13.

36. Schols AM, Creutzberg EC, Buurman WA, et al. Plasma leptin is related to proinflammatory status and dietary intake in patients with chronic obstructive pulmonary disease. Am J Respir Crit Care Med 1999:160:1220-6.

37. Cohen RI, Marzouk K, Berkoski P, et al. Body composition and resting energy expenditure in clinically stable, non-weight-losing patients with severe emphysema. Chest 2003;124:1365-72.

38. Yuan R, Mayo JR, Hogg JC, et al. The effects of radiation dose and CT manufacturer on measurements of lung densitometry. Chest 2007;132:617-23.

39. Okazawa M, Paré PD, Lambert RK. Compliance of peripheral airways deduced from morphometry. J Appl Physiol 2000;89:2373-81.

40. Greaves LJ, Richardson LA. Tobacco use, women, gender, and chronic obstructive pulmonary disease: are the connections being adequately made? Proc Am Thorac Soc 2007:4:675-9. 